\title{
IMMEDIATE SERIAL RECALL OF CHINESE CHARACTERS: A STUDY OF INPUT MODALITY EFFECT
}

\author{
Chih-Wei HUE * \\ National Taiwan University, Taipei, Taiwan \\ David FANG and Kung-Yu HSU \\ Chung-Yuan Christian University, Chung-Li, Taiwan
}

Accepted April 1990

\begin{abstract}
Short-term memory for auditorily and visually presented Chinese characters, varying in frequency and homophonic condition, was studied using an immediate serial-recall task. In experiment 1 , where character frequency and homophonic condition were not controlled, auditory presentation produced better recall than visual presentation on the recency as well as the prerecency part of the serial-position function. In experiment 2 and 3, where character frequency and homophonic condition were controlled, auditory presentation produced better recall on the recency part of the serial-position function than visual presentation (a modality effect), and visual presentation produced better recall on the prerecency part of the serial-position function than auditory presentation (a reverse modality effect).
\end{abstract}

It is a well-established fact that auditory presentation of alphabetical materials produces better performance during immediate serial recall on the recency part of the serial-position function than visual presentation (e.g., Conrad and Hull 1968; Corballis 1966; Murry 1966). This finding has stimulated many researchers to investigate the mechanism underlying this 'modality effect' (e.g., Campbell and Dodd 1980; Crowder 1971, 1983; Crowder and Morton 1969; Gardiner 1983; Greene and Crowder 1984), and the result of their research has greatly improved our understanding of human memory (cf. Nairne 1988).

* We thank Yung-Cheng Shen for help in data analysis, and Jcroen G.W. Raaijmakers, Robert G. Crowder and Graham J. Hitch for useful comments on earlier drafts of this article.

Request for reprints should be sent to Chih-Wei Hue, Dep. of Psychology, National Taiwan University, Taipei, Taiwan, Republic of China. 
In a recent study, Tzeng and Wang (1983) investigated the modality effect by presenting English words to native English speakers and Chinese characters to native Chinese speakers. (Wang (1973) provided an excellent and concise introduction of the written Chinese language.) Classical modality effects were obtained for both groups of subjects. Auditory presentation of words and characters produced greater recency effects than visual presentation. In addition, a 'reverse modality effect' was obtained for the character condition. Visual presentation of character lists produced better recall on the prerecency part of the serial-position function than auditory presentation, whereas no such effect was found for word lists.

These results have some implications concerning the processes involved in word recognition and human memory. For example, Tzeng and Wang (1983) speculated that the influence of sensory characteristics of stimuli may not be restricted to the early stages of information processing, and that different memory mechanisms may be involved in the processing of different types of written materials. More visual memory may be involved in processing Chinese characters than in processing English words.

Before engaging in too much theoretical speculation, one should make sure that the reverse modality effect is a repeatable finding in immediate serial recall of Chinese characters. Questions can be asked concerning the stimuli used in Tzeng and Wang's (1983) experiment. Most Chinese charactcrs have a one-syllable pronunciation; however, many characters are exact homophones. In one popular dictionary, The Chinese Character Dictionary (1985), more than 11,000 characters are listed, but only 1,310 distinctive pronunciations (including tone) are given. Thus, on average, each pronunciation is associated with 10 different characters. However, this may not be an unbiased estimate for the 'internal' pronunciation to character ratio in one's mental lexicon, because literate Chinese are estimated to know only about 5,000 characters. In norms prepared by the National Institute for Compilation and Translation, which rank about 5,000 characters by their linguistic frequencies, 953 distinctive pronunciations are given. Thus, in one's lexicon, each pronunciation is associated with about five characters on average.

It can be seen that without careful control, homophones can be easily included in a stimulus list, and influence memory performance in two possible ways other than Tzeng and Wang's suggestion. First, when 
a pronunciation is heard, the characters associated with the pronunciation are activated. As a result, the number of characters activated during list presentation is more than the number of characters presented. 'This may be the reason why Tzeng and Wang (1983) found that auditory presentation of Chinese characters produces lower memory performance than visual presentation. The second possible result is that pronunciations activate only the high-frequency characters. This should produce better recall for auditory rather than visual presentation, due to a frequency effect.

Since no replication of Tzeng and Wang's (1983) data has been reported, in the present study we investigated immediate serial recall of auditorily and visually presented Arabic numerals, Chinese number characters, and other common Chinese characters. Experiment 1 replicated Tzeng and Wang's (1983) experiment by studying immediate serial recall of common characters selected randomly from the National Institute character norms. In experiment 2 which used Arabic numerals and Chinese number characters as stimuli and in experiment 3 which used homophones and monophonic characters as stimuli, the effects of homophonic condition on immediate serial recall were investigated.

\section{Experiment 1}

Experiment 1 was intended to demonstrate the reverse modality effect using Chinese characters as stimuli. Subjects were required to memorize auditorily or visually presented characters, and after the final character, to recall these characters immediately in their input order.

\section{Method}

\section{Subject}

Twenty-four students from introductory psychology classes at the Chung-Yuan Christian University participated to fulfill a course requirement.

\section{Materials}

Twenty-four lists, each consisting of nine characters randomly selected from the National Institute character norms, were constructed. Each character occurred once in the experiment. The mean and standard deviation of the frequency rank of these characters were $2,340.00$ and 866.20 , respectively.

The stimuli were prepared in two ways. For the visual condition, each character was handwritten on an index card. For the auditory condition, the pronunciations of the characters in a list were tape recorded. 


\section{Procedure}

Subjects in the experiments reported here were tested in groups of three to five at a time. All subjects in this experiment received 12 randomly selected lists presented auditorily and 12 lists presented visually. The first two lists of each condition were used as practice lists. Half of the subjects received auditory lists before visual lists, and the other half received visual lists before auditory lists.

Each trial began with a warning signal, followed by the nine items presented either in auditory or visual format. These items were presented in a sequence, at a rate of 500 msec per item. After the last item was presented, subjects had to write down the list items by their input order on a response sheet.

\section{Results and Discussion}

All significance tests in experiments reported here were performed on the probabilities of correct recall. The definition for correct recall is different for the two presentation conditions. In the visual condition, a response was correct only if it was the correct input character and at the correct position. In the auditory condition, a response was correct if its pronunciation was the same as the input character and at the correct input position. A statistical significance level of 0.05 was used in experiments reported here.

List items were partitioned into two sub-sets: recency set (items presented at the last two serial positions) and prerecency set (the other items). The difference in probability of recall of the recency items between the auditory and the visual conditions was used as measure of modality effect, and the difference in probability of recall of the prerecency items between the two presentation conditions was used as measure of reverse modality effect.

As shown in fig. 1, auditory presentation produced better recall than visual presentation at each serial position. Analysis of the recency items revealed that auditory presentation produced better performance than visual presentation $(F(1,23)=$ 93.23, $M S \mathrm{e}=0.0408$ ), the mean probabilities of recall were 0.62 and 0.22 , respectively, which indicates that a modality effect was obtained in this experiment. Analysis of the prerecency items also revealed that auditory presentation produced better performance than visual presentation $(F(1,23)=26.58, M S \mathrm{e}=0.0597)$, the mean probabilities of recall were 0.47 and 0.33 , respectively.

Although, a modality effect was found in this experiment, the pattern of the results was different from that found in Tzeng and Wang's (1983) experiment. The present experiment failed to obtain a reverse modality effect in immediate serial recall of Chinese characters.

A possible reason for our failure to replicate Tzeng and Wang's (1983) results was that in the auditory condition, the pronunciations of the characters in a list did not activate the characters themselves, but activated the homophones of the characters, which have higher frequency. Thus, the average frequency of the characters activated in the auditory condition was higher than that of the characters activated in the visual condition. The superior performance for the auditory condition can be interpreted in terms of a frequency effect. Analysis of the correct responses in the auditory condition 


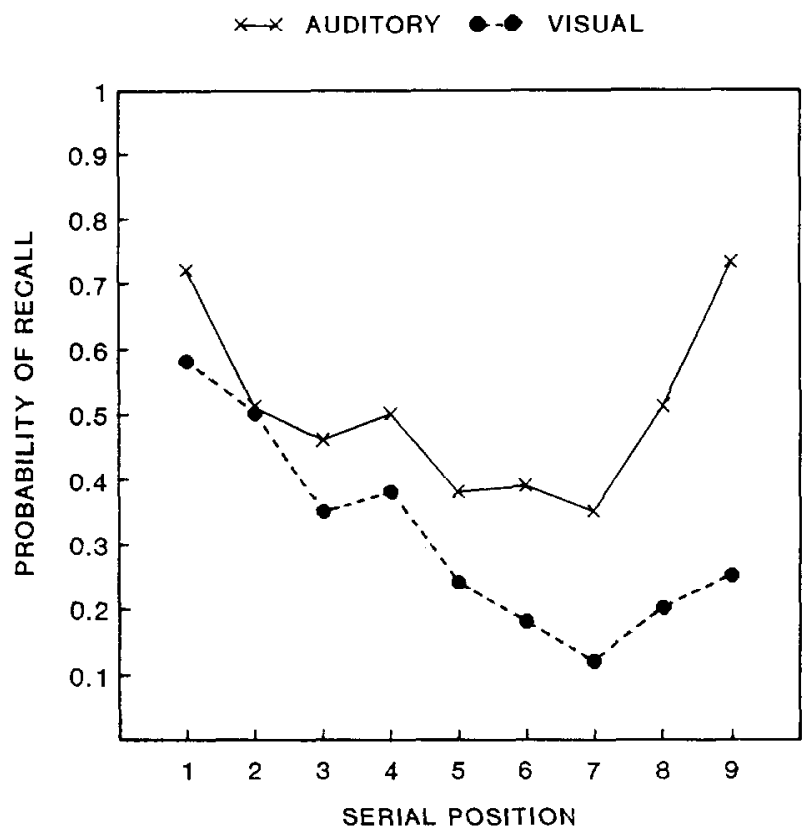

Fig. 1. Data from experiment 1: mean probabilities of recall as functions of serial position and presentation condition.

provided support for this interpretation. The mean frequency rank of these responses was 1474.80 , which was considerably higher than that of the stimulus characters.

\section{Experiment 2}

To control the possible frequency confounding found in experiment 1 , Arabic numerals and Chinese number characters were used as stimuli in this experiment. In this context, the items in the auditory condition could not be interpreted otherwise but as numbers.

\section{Method}

\section{Subjects}

Twenty-four students from introductory psychology classes at the National Taiwan University participated to fulfill a course requirement.

\section{Materials}

Thirty-six lists, each consisting of the 10 digits from zero to nine, were constructed. In each list, the 10 digits were randomly ordered. The lists were prepared in three ways. 
In the Arabic numeral condition, each digit was handwritten on an index card in Arabic numerals, in the character condition, each digit was hand written on an index card in Chinese characters, and in the auditory condition, the pronunciations of the digits of a list were tape recorded.

\section{Procedure}

All subjects received 12 randonly selected lists presented in Arabic numeral format, 12 in character format, and 12 in auditory format. The first two lists of each condition were used as practice lists. Each trial began with a warning signal, followed by the 10 items presented at a rate of $500 \mathrm{msec}$ per item. After the presentation of the last item. subjects had to write down these items. In the Arabic numeral condition, subjects responded with Arabic numerals. In the Chinese character and the auditory conditions, subjects responded with Chinese characters.

\section{Results and Discussion}

The results are given in fig. 2 and show all of the important characteristics of the standard modality effect and the reverse modality effect.

Analysis of the recency items revealed that auditory presentation produced better performance than visual presentation of Arabic numerals and number characters

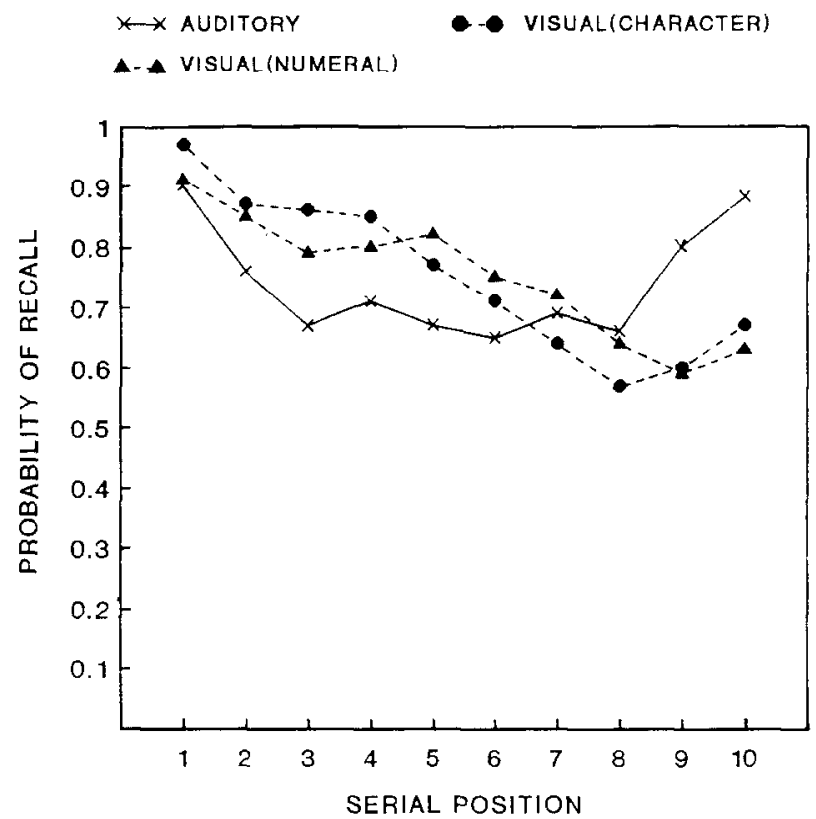

Fig. 2. Data from experiment 2: mean probabilities of recall as functions of serial position and presentation condition. 
$(F(2,46)=16.47, M S \mathrm{c}=0.0466)$, the mean probabilities of recall were $0.84,0.61$, and 0.64 , respectively. Analysis of prerecency items revealed opposite effects. Visual presentation of Arabic numerals and number characters produced better performance than auditory presentation $(F(2,46)=4.09, M S e=0.0732)$, the mean probabilities of recall were $0.78,0.78$, and 0.71 , respectively. That is, a reverse modality effect was obtained.

\section{Experiment 3}

Experiment 3 investigated further the hypothesis that the failure to find reverse modality effects in experiment 1 was caused by a frequency confounding in the auditory condition. In this experiment, high- and low-frequency homophones and monophonic characters were used as stimuli. If subjects selectively associate auditory items with high-frequency characters, then auditory presentation of low-frequency homophones should produce better performance than visual presentation of these items on the recency as well as the prerecency positions of the serial-position function. On the other hand, reverse modality effects should be obtained for the other conditions.

\section{Method}

\section{Subjects}

Thirty-two students from introductory psychology classes at the National Taiwan University participated to fulfill a course requirement. Sixteen subjects participated in the auditory condition, and 16 participated in the visual condition.

\section{Materials and procedure}

Four sets of characters were selected from the National Institute character norms by crossing homophonic condition (homophones or monophonic characters) and frequency (high or low). The mean frequency ranks for high-frequency homophones and monophonic characters and for low-frequency homophones and monophonic characters were $456.70,482.50,1,832.70$, and $1,622.00$, respectively. The mean frequency rank of the characters which has the highest frequency rank associatcd with the pronunciations of the low-frequency homophones was 370.00 .

Each character set contained 54 characters and was randomly divided into six 9-item lists. In addition, four lists, each containing nine common characters, were selected for practice lists. The stimuli were prepared in two ways, tape recorded for the auditory condition and handwritten for the visual condition.

After the four practice lists were administered, the 24 stimuli lists were presented in random order.

\section{Results and Discussion}

The results are shown in fig. 3. Four separate analyses were performed for high- and low-frequency homophones and monophonic characters. For the high-frequency homo- 
$\longleftrightarrow x$ AUDITORY - VISUAL

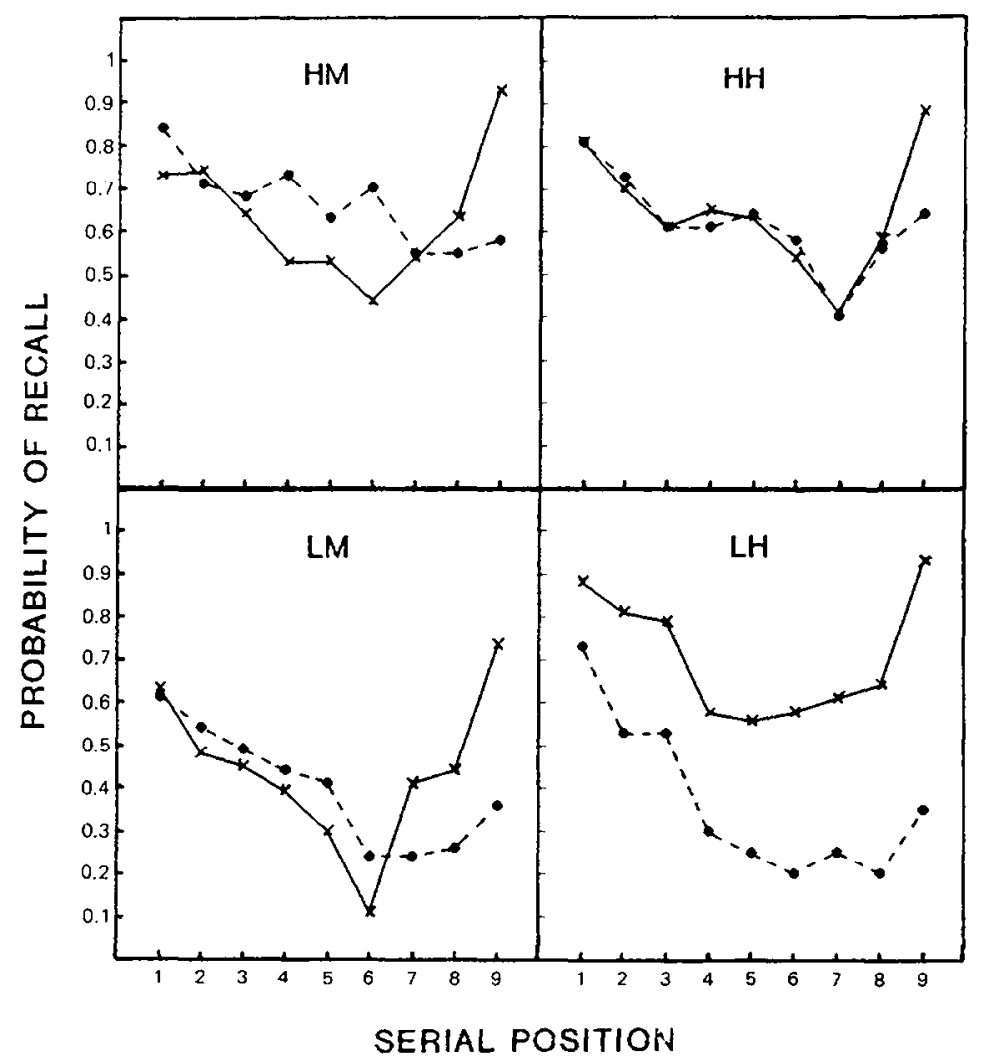

Fig. 3. Data from experiment 3: mean probabilities of recall in each type of character list as functions of serial position and presentation condition.

phonic lists, analysis of recency items showed a non-significant trend of modality effect $(F(1,30)=1.56, M S e=0.1682, p=0.22)$. The mean probabilities of recall for the auditory and the visual conditions were 0.73 and 0.60 , respectively.

For the low-frequency homophonic lists, analyses of the recency items revealed a clear modality effect $(F(1,30)=61.86, M S \mathrm{e}=0.0663)$. The mean probabilities of recall for the auditory and the visual conditions were 0.78 and 0.28 , respectively. Similar effects were also found for the prerecency items $(F(1,30)=26.41, M S e=0.1775)$. The mean probabilities of recall for the auditory and the visual conditions were 0.62 and 0.31 , respectively.

For the high-frequency monophonic character lists, analysis of recency items revealed a modality effect $(F(1,30)=8.55, M S e=0.0845)$. The mean probabilities of recall for the auditory and the visual conditions were 0.78 and 0.56 , respectively. 
Analysis of prerecency items showed a marginally significant reverse modality effect $(F(1,30)=3.12, M S \mathrm{e}=0.1797, p=0.08)$. The mean probabilities of recall for the auditory and the visual conditions were 0.69 and 0.59 , respectively.

For the low-frequency monophonic character lists, analysis of recency items revealed a modality effect $(F(1,30)=13.31, M S e=0.0868)$. The mean probabilities of recall for the auditory and the visual conditions were 0.58 and 0.31 , respectively. The effect of presentation condition was not significant for the prerecency items.

Although the results of the present experiment were not exactly as predicted, the general pattern of the results is consistent with our hypothesis. In each condition, auditory presentation produced a greater recency effect than visual presentation. On the other hand, visual presentation produced better performance on the prerecency part of the serial-position function than visual presentation of the high- and low-frequency monophonic characters. However, for the low-frequency homophones, the pattern of the results was the same as that obtained in experiment 1 . That is, visual presentation produced better performance than auditory presentation at the recency as well as the prerecency positions of the serial-position function.

\section{General discussion}

The results of the present research show modality and reverse modality effects in immediate serial recall of Chinese characters. Because homophones are prevalent in Chinese, an attempt was made to investigate its effects on recall.

Although it is a common belief that the primary coding for processing Chinese characters is visual (e.g., Rozin et al. 1971; Sasanuma 1975), on the basis of the three experiments reported here, there is reason to believe that homophonic condition should be controlled in studies of memory of Chinese characters. In experiment 1 , where homophonic condition was not controlled, auditory presentation produced better recall than visual presentation on the recency as well as the prerecency part of the serial-position function. In experiments 2 and 3, where homophonic condition was controlled, auditory presentation produced better recall on the recency part of the serial-position function, on the other hand, visual presentation produced better recall on the prerecency part of the function.

Many theories have been proposed as explanations for the modality effect. One prominent account suggests that auditory sensory memory, also called precategorical acoustic storage (PAS), has greater durability compared with visual sensory memory (Crowder and Morton 1969). Recently, the PAS account has been challenged by new findings (e.g., 
Campbell and Dodd 1980; Nairne and Walters 1983; Shand and Klima 1981 ), and many new explanations have been proposed (see Nairne (1988) for reviews). For example, Glenberg (1984) proposed that there is less potential for extra experimental stimuli to interfere with auditory memory traces than with visual traces following list presentation. As a result, auditory presentation produces better recall on the recency part of the serial-position function. Alternatively, Shand and Klima (1981) proposed that the dominant coding format in short-term memory is acoustic, and that recency effects result when the presentation format is consistent with the subjects' dominant coding format in short-term memory. Apparently, none of these interpretations can adequately account for the data reported here.

It is possible that there may not be a common mechanism underlying the modality and the reverse modality effect reported in the present research. It has been demonstrated that Chinese speakers' dominant coding in short-term memory is verbal (e.g., Tzeng et al. 1977), hence, substantial recency effects result when the presentation mode is auditory. The reverse modality effect may be accounted for by using a concept similar to that proposed by Glenberg (1984). Visual codes are more distinct than auditory codes in the processing of Chinese language. It has been noted above that there are many homophones in Chinese, so that auditory codes easily overlap with each other, especially for prerecency items. On the other hand, because of their distinctiveness, visual codes are less likely to interfere with other visual codes.

Although the mechanism involved in producing modality and reverse modality effects is far from clear, Chinese characters are promising as stimuli for experiments designed to answer the question. In a recent article, Nairne (1988) argued that immediate serial recall is a reconstructive process involving the analysis of multi-attribute memory traces formed during list presentation. These traces are primarily of two types, modality-independent traces resulting from internal verbalization of the inputs and modality-dependent traces which consist of those physical and intra-item features that are unique to the particular mode of presentation. Chinese characters provide different modalitydependent features than alphabetic materials, making them very useful as stimulus materials to investigate human memory. 


\section{References}

Campbell, R., and B. Dodd, 1980. Hearing by eye. Quarterly Journal of Experimental Psychology $32,85-99$.

Conrad, R. and A. J. Hull, 1968. Input modality and the serial position curve in short-term memory. Psychonomic Science, 10, 135-136.

Corballis, M.S., 1966. Rehearsal and decay in immediate recall of visually and aurally presented items. Canadian Journal of Psychology 20, 43-51.

Crowder, R.G., 1971. The sounds of vowels and consonants in immediate memory. Journal of Verbal Learning \& Verbal Behavior 10, 587-596.

Crowder, R.G., 1983. The purity of auditory memory. Philosophical Transactions of the Royal Society of London 302B, 251-265.

Crowder, R.G. and J. Morton, 1969. Precategorical acoustic storage (PAS). Perception \& Psychophysics 5, 365-373.

Far East Chinese Character Dictionary, 1985. Taipei: Far East.

Gardiner, J.M., 1983. On recency and echoic memory. Philosophical Transactions of the Royal Society of London 302B, 267-282.

Glenberg, A.M., 1984. A retrieval account of the long-term modality effect. Journal of Experimental Psychology: Learning, Memory, and Cognition 9, 231-255.

Greene, R.L. and R.G. Crowder, 1984. Modality and suffix effects in the absence of auditory stimulation. Journal of Verbal Learning \& Verbal Behavior 23, 371-382.

Murry, D.J., 1966. Vocalization-at-presentation and immediate recall, with varying recall methods. Quarterly Journal of Experimental Psychology 15, 9-18.

Nairne, J.S., 1988. A framework for interpreting recency effects in immediate serial recall. Memory and Cognition 16, 343-352.

Nairne, N.S. and V.L. Walters, 1983. Silent mouthing produces modality- and suffix-like effects. Journal of Verbal Learning \& Verbal Behavior 22, 475-483.

National Institute for Compilation and Translation, 1967. A study on the high frequency in Chinese elementary school reading materials. Taipei: Chung Hwa.

Rozin, P., S. Poritsky and R. Sotsky, 1971. American children with reading problems can easily learn to read English represented by Chinese characters. Science 171, 1264-1267.

Sasanuma, S., 1975. Kana and Kanji processing in Japanese aphasics. Brain and Language 3, 141-157.

Shand, M.D. and E.S. Klima, 1981. Nonauditory suffix effects in congenitally deaf signers of American sign language. Journal of Experimental Psychology: Human Learning Memory 7 , $464-474$.

Tzeng, O.J.L. and W.S.-Y. Wang 1983. The first two R's. American Scientist 71, 238-243.

Tzeng, O.J.L., D.L. Hung and W.S.-Y. Wang, 1977. Speech recoding in reading Chinese characters. Journal of Experimental Psychology: Human Learning and Memory 3, 621-630.

Wang, W.S.-Y., 1973. The Chinese language. Scientific American 228, 51-60. 